\title{
A METHOD OF FRACTIONAL STEPS FOR SCALAR CONSERVATION LAWS WITHOUT THE CFL CONDITION
}

\author{
HELGE HOLDEN AND NILS HENRIK RISEBRO
}

\begin{abstract}
We present a numerical method for the $n$-dimensional initial value problem for the scalar conservation law $u\left(x_{1}, \ldots, x_{n}, t\right)_{t}+\sum_{i=1}^{n} f_{i}(u)_{x_{i}}=0$, $u\left(x_{1}, \ldots, x_{n}, 0\right)=u_{0}\left(x_{1}, \ldots, x_{n}\right)$. Our method is based on the use of dimensional splitting and Dafermos's method to solve the one-dimensional equations. This method is unconditionally stable in the sense that the time step is not limited by the space discretization. Furthermore, we show that this method produces a subsequence which converges to the weak entropy solution as both the time and space discretization go to zero. Finally, two numerical examples are discussed.
\end{abstract}

\section{INTRODUCTION}

Scalar conservation laws, given their wide range of applications, have been studied extensively over the years, both from a mathematical, physical and numerical point of view. Fundamental problems are the emergence of discontinuous solutions of the partial differential equation with the subsequent call for weak solutions, which again results in subtle uniqueness questions. Existence and uniqueness were first proved for the general Cauchy problem by Conway and Smoller [2], and later on by Kuznetsov [9], Vol'pert [10], Kružkov [8] who used a viscosity method. We will here use Kružkov's formulation of the entropy condition, which is a mechanism to identify the unique physical solution.

We here study the Cauchy problem

$$
\begin{gathered}
u_{t}+\sum_{i=1}^{n} f_{i}(u)_{x_{i}}=0, \\
u\left(x_{1}, \ldots, x_{n}, 0\right)=u_{0}\left(x_{1}, \ldots, x_{n}\right) .
\end{gathered}
$$

Kružkov's definition of the entropy weak solution reads as follows: $u$ is the entropy weak solution if for all constants $k$, all $\phi \in C_{0}^{1}, \phi \geq 0$, the inequality

Received by the editor August 27, 1991 and, in revised form, January 28, 1992.

1991 Mathematics Subject Classification. Primary 35L65, 65M12, 65M99.

Key words and phrases. Dimensional splitting, scalar conservation law, fractional steps, numerical methods.

We are grateful to Frode Bratvedt and Technical Software Consultants for permission to use their software. 


$$
\begin{array}{r}
\int_{\mathbb{R}^{n}} \int_{t>0}\left[\phi_{t}|u-k|+\right. \\
\left.\operatorname{sign}(u-k) \sum_{i=1}^{n}\left(f_{i}(u)-f_{i}(k)\right) \phi_{x_{i}}\right] d^{n} x d t \\
+\int_{\mathbb{R}^{n}}\left|u_{0}-k\right| \phi\left(x_{1}, \ldots, x_{n}, 0\right) d^{n} x \geq 0
\end{array}
$$

holds.

The method of fractional steps, or dimensional splitting, was introduced by Godunov [5] in connection with gas dynamics, and later modified and extended by various authors.

Let us briefly describe the method of fractional steps, due to Godunov, for the case $n=2$. Let $u(x, y, t)=S(t) u_{0}(x, y)$ denote the entropy solution of

$$
\begin{gathered}
u_{t}+f(u)_{x}+g(u)_{y}=0 \\
u(x, y, 0)=u_{0}(x, y)
\end{gathered}
$$

at time $t$. Similarly, let $v(x, y, t)=S^{f, x}(t) v_{0}(x, y)$ denote the entropy solution of

$$
\begin{gathered}
v_{t}+f(u)_{x}=0, \\
v(x, y, 0)=v_{0}(x, y)
\end{gathered}
$$

at time $t$, when $y$ is considered a parameter. The idea is then to alternately apply the operators $S^{f, x}$ and $S^{g, y}$ (defined as $S^{f, x}$, but with $y$ as a parameter) for small time steps $\Delta t$ to approximate $u(x, y, t)$, viz.,

$$
u(x, y, t)=\left(S(t) u_{0}\right)(x, y) \approx\left[S^{f, x}(\Delta t) S^{g, y}(\Delta t)\right]^{n} u_{0}(x, y)
$$

with $n \Delta t=t$.

When solving the one-dimensional problem $(0.5)$, one may choose from a variety of methods available. Crandall and Majda [3] analyze rigorously the method of fractional steps for monotone schemes, the Glimm method, and the Lax-Wendroff scheme.

We here propose another scheme which has the advantage of yielding an unconditionally stable approximation in the sense that the time step is not limited by the space step used in the discretization; i.e., one does not need the CourantFriedrichs-Lewy (CFL) condition. Our method is based on an idea by Dafermos [4] of approximating the flux function by a polygon, i.e., a continuous, piecewise linear function. Furthermore, the initial data are approximated by step functions, thereby yielding (multiple) Riemann problems. This has the advantage of replacing rarefaction waves by shocks in the solution, and thus the solution will be a step function in $x$ for each $t$. Holden, Holden, and Høegh-Krohn [6], [7] developed this approach into a numerical method for $n=1$.

We now give a brief summary of the paper. Let $\delta>0$ denote the parameter measuring the polygonal approximation of the flux function in the sense of (1.2), and fix a grid in the $x, y$-plane. We then use the Dafermos scheme in the $x$-direction for a small time step $\Delta t$. The solution is then projected back onto the original grid before we apply the Dafermos scheme in the $y$-direction for a time step $\Delta t$, using the solution computed in the $x$-direction as initial data. Each time after we apply the Dafermos scheme, we project the function onto the original grid, thereby obtaining a sequence of functions indexed by the 
number of iterations and the mesh size. In a series of lemmas we then prove that this sequence is uniformly bounded by the initial data in the $L^{\infty}$-norm, the T.V.-norm, and has $L_{1}$-norm which is Lipschitz continuous in the time variable. Helly's theorem then gives a convergent subsequence, which is finally proved to satify the Kružkov entropy condition (0.2). In a last section we apply this method to two problems in two dimensions, one theoretical example and one example taken from petroleum reservoir simulation.

\section{Construction of APPROXimate solutions}

For simplicity of notation we will consider $(0.1)$ in two dimensions, since generalization to more than two dimensions is straightforward. In two dimensions, (0.1) reads

$$
\begin{gathered}
u_{t}+f(u)_{x}+g(u)_{y}=0, \\
u(x, y, 0)=u_{0}(x, y),
\end{gathered}
$$

where $f$ and $g$ are continuous functions $\mathbb{R} \rightarrow \mathbb{R}$ that are also in $B V_{\text {loc }}(\mathbb{R}) \cap$ $L_{\text {loc }}^{1}(\mathbb{R})$.

We wish to construct a numerical approximation of the solution $u$ based on dimensional splitting, and where the one-dimensional solution operators are constructed by Dafermos's method [4]. We now give a brief description of Dafermos's method as used in [7] and as we will use it here.

Let $u_{0}$ be some given real number and let $u_{i}=u_{0}+i \delta, \delta>0$, for $i=$ $1 \ldots, N$; let $f_{i}=f\left(u_{i}\right)$. We then define $f_{\delta}(u)$ by

$$
u \in\left[u_{i}, u_{i+1}\right] \Rightarrow f_{\delta}(u)=\frac{f_{i+1}-f_{i}}{u_{i+1}-u_{i}}\left(u-u_{i}\right)+f_{i}, \quad i=0, \ldots, N-1
$$

and

$$
u \leq u_{0} \Rightarrow f_{\delta}(u)=u_{0}, \quad u \geq u_{N} \Rightarrow f_{\delta}(u)=u_{N} .
$$

Consider the Riemann problem with $u_{l}=u_{0}$ and $u_{r}=u_{N}$. Let $f_{c}$ denote the lower convex envelope of $f_{\delta}$ on $\left[u_{l}, u_{r}\right]$. Then also $f_{c}$ is piecewise linear and continuous. Let $\tilde{u}_{0}<\tilde{u}_{1}<\cdots<\tilde{u}_{M}$ be such that

$$
\tilde{u}_{0}=u_{0}, \quad \tilde{u}_{M}=u_{N}, \quad\left\{\tilde{u}_{0}, \ldots, \tilde{u}_{M}\right\} \subseteq\left\{u_{0}, \ldots, u_{N}\right\},
$$

and such that $f_{c}$ is linear on each interval $\left[\tilde{u}_{i}, \tilde{u}_{i+1}\right]$. The solution of the onedimensional Riemann problem with left state $u_{0}$ and right state $u_{N}$ is now given by

$$
u(x, t)= \begin{cases}u_{l} \text { for } x \leq \tilde{s}_{0} t \\ \tilde{u}_{i} \text { for } \tilde{s}_{i-1} t<x \leq \tilde{s}_{i} t, i=1, \ldots, M-1, \\ u_{r} \text { for } x>\tilde{s}_{M-1} t\end{cases}
$$

where

$$
\tilde{s}_{i}=\frac{\tilde{f}_{i+1}-\tilde{f}_{i}}{\tilde{u}_{i+1}-\tilde{u}_{i}}, \quad i=0, \ldots, M-1 .
$$

There is a similar formula involving the upper convex envelope for the solution of the Riemann problem in the case where the left initial value is larger than the right. In particular, we see that the solution in each case is a step function in $x / t$. Dafermos's method as used in [7] and elsewhere involves approximating 
the initial function by a step function and thereby defining a series of Riemann problems. The solutions of these will define a function which can be defined for $t>0$ until two discontinuities interact. The interacting discontinuities will then define a Riemann problem. This Riemann problem is solved and the solution can be continued in this fashion up to any positive time. For a complete description of this procedure we refer the reader to [6], [7].

Let $\Delta x$ and $\Delta y$ be given (small) numbers, and let $\pi$ be a projection from $B V\left(\mathbb{R}^{2}\right)$ to functions that are constant on each square

$$
z_{i j}=\{(x, y) ; i \Delta x<x<(i+1) \Delta x, j \Delta y<y<(j+1) \Delta y\}
$$

for $i, j \in \mathbb{Z}$. The projection $\pi$ is to satisfy

$$
\begin{gathered}
\lim _{\Delta x \rightarrow 0, \Delta y \rightarrow 0} \pi u(x, y)=u(x, y), \\
\iint|\pi u-u| d x d y=O(\max (\Delta x, \Delta y)), \\
(\pi u)_{i j} \Delta x \Delta y=\int_{i \Delta x}^{(i+1) \Delta x} \int_{j \Delta y}^{(j+1) \Delta y} \pi u d x d y=\int_{i \Delta x}^{(i+1) \Delta x} \int_{j \Delta y}^{(j+1) \Delta y} u d x d y,
\end{gathered}
$$

where we write $(\pi u)_{i j}$ for $\left.\pi u\right|_{z_{i j}}$. Furthermore, the value of $\pi u$ in $z_{i j}$ should only depend on $u$ in $z_{i j}$. In addition, the projection is required to satisfy $\min _{(x, y) \in z_{i j}} u \leq(\pi u)_{i j} \leq \max _{(x, y) \in z_{i j}} u$.

The canonical choice would be to let $\pi$ denote the grid average, i.e.,

$$
\pi u(x, y)=\mu\left(z_{i j}\right)^{-1} \int_{z_{i j}} d \mu(\tilde{x}, \tilde{y}) u(\tilde{x}, \tilde{y}), \quad(x, y) \in z_{i j},
$$

for some measure $\mu$. Since we will use Dafermos's method in each direction, we define $f_{\delta}$ and $g_{\delta}$ to be piecewise linear continuous approximations to $f$ and $g$, respectively. The approximations should be good both in the T.V.-norm and in $L_{1}$, i.e.,

$$
\begin{gathered}
\lim _{\delta \rightarrow 0}\left|f(u)-f_{\delta}(u)\right|_{\text {T.v. }}=0, \\
\lim _{\delta \rightarrow 0}\left|f(u)-f_{\delta}(u)\right|_{L_{1}}=0
\end{gathered}
$$

and similarly for $g$. If $v_{0}(x)$ is a piecewise constant function taking a finite number of values, we can use Dafermos's method to calculate the solution to the initial value problem

$$
v_{t}+f_{\delta}(v)_{x}=0, \quad v(x, 0)=v_{0}(x) .
$$

We will write $v(x, t)=S_{\delta}^{f, x}(t) v_{0}(x)$ to indicate that $v(x, t)$ is the weak entropy solution of (1.11).

If, for each fixed $x, u(x, y)$ is a piecewise constant function in $y$ on the intervals $\langle j \Delta y,(j+1) \Delta y\rangle, j \in \mathbb{Z}$, we write

$$
u_{j}(x)=\left.u\right|_{j \Delta y<y<(j+1) \Delta y}(x, y) \text {. }
$$

Similarly,

$$
u_{i}(y)=\left.u\right|_{i \Delta x<x<(i+1) \Delta x}(x, y)
$$

for functions that are constant in $x$ for each $y$. Furthermore,

$$
u_{j}(x, t)=S_{\delta}^{f, x}(t) u_{j}(x), \quad u_{i}(y, t)=S_{\delta}^{g, y}(t) u_{i}(y) .
$$


Dimensional splitting consists in first applying the solution operator $S_{\delta}^{f, x}$ to $u_{j}$ for each $j$, then projecting the solution back onto the grid, and subsequently applying the solution operator $S_{\delta}^{g, y}$ to $u_{i}$ for each $i$. Finally, the result of this is projected onto the grid, and the process is repeated. In "computer code", this looks as follows:

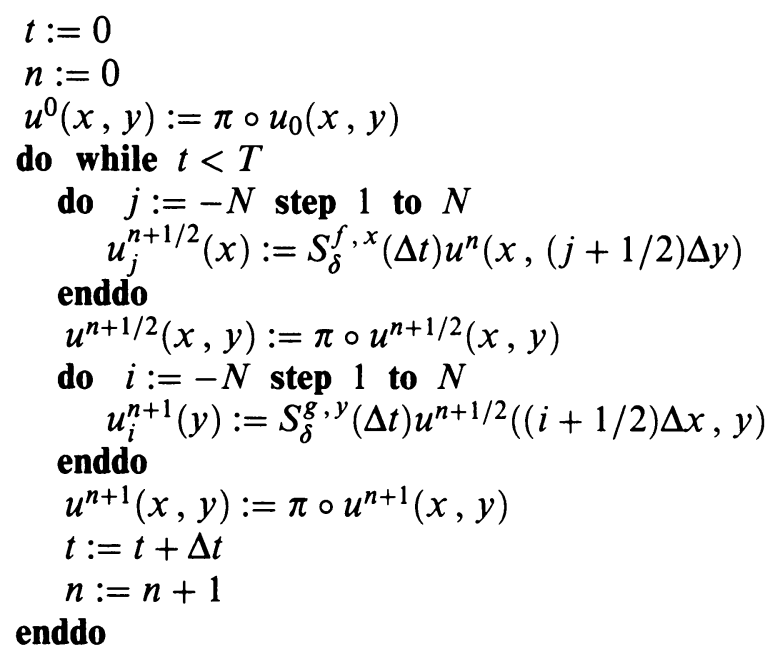

Here, $N$ is a constant that is chosen so large that $u^{n}$ is constant outside the square bounded by $\pm N \Delta x$ and $\pm N \Delta y$ in the time interval $[0, T]$.

\section{Convergence}

For convenience we will from now on assume that $\Delta x=\Delta y=c \Delta t$ for some $c \neq 0$. We then have three main lemmas, which ensure the existence of a convergent subsequence.

\section{Lemma 1. There holds}

$$
\left\|u^{n}(x, y)\right\|_{\infty} \leq\left\|u_{0}(x, y)\right\|_{\infty}
$$

Proof. This is true since $S_{\delta}^{f, x}$ and $S_{\delta}^{g, y}$ do not introduce new maxima or minima, and neither does the projection $\pi$.

Lemma 2. We have

$$
\text { T.V. } \cdot(x, y)\left(u^{n}(x, y)\right) \leq \mathrm{T} . \mathrm{V}_{\cdot(x, y)}\left(u_{0}(x, y)\right) .
$$

Proof. Recall that for a function $h(x, y)$, the total variation T.V. ${ }_{(x, y)} h(x, y)$ is defined as

$$
\mathrm{T} . \mathrm{V} \cdot(x, y) h(x, y)=\int \mathrm{T} . \mathrm{V} \cdot x(h(x, y)) d y+\int \mathrm{T} . \mathrm{V} \cdot y(h(x, y)) d x .
$$

The lemma will hold inductively if we show that

$$
\text { T.V. }\left(u^{n+1}(x, y)\right) \leq \text { T.V. }\left(u^{n}(x, y)\right) \text {. }
$$

From [7] we know that if $u$ and $v$ are two weak solutions of

$$
u_{t}+f(u)_{x}=0
$$

with initial values $u_{0}$ and $v_{0}$, respectively, then 


$$
\int|u-v| d x \leq \int\left|u_{0}-v_{0}\right| d x .
$$

We now have that $u_{j}^{n}(x, \Delta t)$ and $u_{j+1}^{n}(x, \Delta t)$ are step functions that are constant on some intervals $\left\{\left[x_{k}, x_{k+1}\right\rangle\right\}$. Thus, if $\tilde{x}_{k} \in\left[x_{k}, x_{k+1}\right\rangle$, then

$$
\begin{aligned}
& \int\left|u_{j+1}^{n}(x, \Delta t)-u_{j}^{n}(x, \Delta t)\right| d x \\
& \quad=\sum_{k}\left|u_{j+1}^{n}\left(\tilde{x}_{k}, \Delta t\right)-u_{j}^{n}\left(\tilde{x}_{k}, \Delta t\right)\right|\left(x_{k+1}-x_{k}\right) \\
& \quad \leq \sum_{i}\left|u_{i, j+1}^{n}-u_{i, j}^{n}\right| \Delta x,
\end{aligned}
$$

using (2.5). But by the construction of the projection $\pi$,

$$
\sum_{k}\left|u_{j+1}^{n}\left(\tilde{x}_{k}, \Delta t\right)-u_{j}^{n}\left(\tilde{x}_{k}, \Delta t\right)\right|\left(x_{k+1}-x_{k}\right)=\sum_{i}\left|u_{i, j+1}^{n+1 / 2}-u_{i, j}^{n+1 / 2}\right| \Delta x .
$$

Therefore,

$$
\sum_{i}\left|u_{i, j+1}^{n+1 / 2}-u_{i, j}^{n+1 / 2}\right| \Delta x \leq \sum_{i}\left|u_{i, j+1}^{n}-u_{i, j}^{n}\right| \Delta x .
$$

If again $u$ is a weak solution of (2.4), then from [6] we have

$$
\text { T.V.x }(u) \leq \text { T.V.x }\left(u_{0}\right) \text {. }
$$

From this it follows that

$$
\sum_{k}\left|u_{j}^{n}\left(\tilde{x}_{k+1}, \Delta t\right)-u_{j}^{n}\left(\tilde{x}_{k}, \Delta t\right)\right| \leq \sum_{i}\left|u_{i+1, j}^{n}-u_{i, j}^{n}\right| .
$$

Now let $h=h(x) \in B V$ be any piecewise constant function, and let $h_{c}$ be a continuous approximation to $h$ defined as follows. In a small neighborhood of each jump we let $h_{c}$ be a linear interpolation between the two constant values. Then T.V. $(h)=$ T.V. $\left(h_{c}\right) \geq$ T.V. $(\pi h)$, since $\pi h$ is a particular partition of $h_{c}$. This implies

$$
\sum_{i}\left|u_{i+1, j}^{n+1 / 2}-u_{i, j}^{n+1 / 2}\right| \leq \sum_{i}\left|u_{i+1, j}^{n}-u_{i, j}^{n}\right|
$$

Multiplying (2.11) by $\Delta y$ and summing over $j$, and summing (2.8) over $j$, and then adding the results, we obtain

$$
\mathrm{T} . \mathrm{V} \cdot(x, y)_{\left(u^{n+1 / 2}(x, y)\right) \leq \mathrm{T} . \mathrm{V} \cdot(x, y)}\left(u^{n}(x, y)\right) .
$$

The desired result now follows by applying $S_{\delta}^{g, y}$.

Lemma 3. There holds

$$
\sum_{i, j}\left|u_{i, j}^{m}-u_{i, j}^{n}\right| \Delta x \Delta y=(C \Delta t+h(\Delta x, \Delta y, u))(m-n),
$$

where $h$ is such that $\lim _{\Delta x \rightarrow 0, \Delta y \rightarrow 0} h(\Delta x, \Delta y, u)=0$.

Proof. If we again turn to the one-dimensional equation and let $u$ be the solution of (2.4), then

$$
\int\left|u\left(x, t_{2}\right)-u\left(x, t_{1}\right)\right| d x \leq C\left(t_{2}-t_{1}\right)
$$


for some constant $C$. In our notation this reads

$$
\sum_{k}\left|u_{j}^{n+1 / 2}\left(\tilde{x}_{k+1}, \Delta t\right)-u_{j}^{n}\left(\tilde{x}_{k}\right)\right|\left(x_{k+1}-x_{k}\right) \leq C \Delta t,
$$

where the intervals $\left\{\left[x_{k}, x_{k+1}\right\rangle\right\}$ are chosen such that both $u_{j}^{n}(x, \Delta t)$ and $u_{j}^{n}(x)$ are constant on $\left[x_{k}, x_{k+1}\right\rangle$ and $\tilde{x}_{k} \in\left[x_{k}, x_{k+1}\right\rangle$. Now

$$
\begin{aligned}
\left|u_{i, j}^{n+1}-u_{i, j}^{n}\right| \leq & \left|u_{i, j}^{n+1}-u_{j}^{n}(x, \Delta t)\right|+\left|u_{j}^{n}(x, \Delta t)-u_{i, j}^{n+1 / 2}\right| \\
& +\left|u_{i, j}^{n+1 / 2}-u_{j}^{n+1 / 2}(x, \Delta t)\right|+\left|u_{j}^{n+1 / 2}(x, \Delta t)-u_{i, j}^{n}\right|,
\end{aligned}
$$

for $i \Delta x \leq x<(i+1) \Delta x$. Integrating (2.16) in both the $x$-and $y$-direction and using (2.15) gives

$$
\sum_{i, j}\left|u_{i, j}^{n+1}-u_{i, j}^{n}\right| \Delta x \Delta y \leq 4 N C \Delta t+\iint|\pi v-v| d x d y+\iint|\pi w-w| d x d y
$$

where

$$
\begin{aligned}
& w(x, y)=u_{j}^{n}(x, \Delta t), \\
& v(x, y)=u_{j}^{n+1 / 2}(x, \Delta t),
\end{aligned}
$$

and $N$ is such that $u_{i, j}^{n}$ is constant outside the square bounded by $\pm N \Delta x$ and $\pm N \Delta y$. By virtue of $(1.8)$, the last two terms on the right-hand side of (2.17) will be of order $O(\Delta x)=O(\Delta t)$ as $\Delta x$ and $\Delta y$ tend to zero. The lemma now follows by induction.

Denote $u^{n}(x, y)$ by $u_{\eta}^{n}(x, y)$, where $\eta=(\delta, \Delta x)$. Now by using Lemmas 1-3 and Helly's theorem as, e.g., in [2], one shows the existence of a convergent subsequence of $u_{\eta}^{n}$ (which we for simplicity will again call $u_{\eta}^{n}(x, y, t)$ ). Furthermore, this sequence converges uniformly in $L_{1}\left(\mathbb{R}^{2} \times[0, T]\right)$ for any $T>0$, and the limit takes the correct initial value. We will denote this limit by $u(x, y, t)$.

Lemma 4. The limit $u(x, y, t)$ is a weak entropy solution of (2.1).

Proof. We always have that $u_{j}^{n}(x, \Delta t)$ is a weak entropy solution of the problem

$$
u_{t}+f(u)_{x}=0, \quad u(x, n \Delta t)=u_{j}^{n}(x)
$$

Therefore,

$$
\begin{aligned}
\int_{\mathbb{R}} \int_{n \Delta t}^{(n+1) \Delta t} \phi_{t}\left|u_{j}^{n}(x, t)-k\right| \\
+\phi_{x} \operatorname{sign}\left(u_{j}^{n}(x, t)-k\right)\left(f_{\delta}\left(u_{j}^{n}(x, t)\right)-f_{\delta}(k)\right) d t d x \\
-\int_{\mathbb{R}} \phi(x,(n+1) \Delta t)\left|u_{j}^{n}(x, \Delta t)-k\right| d x \\
+\int_{\mathbb{R}} \phi(x, n \Delta t)\left|u_{j}^{n}(x)-k\right| d x \geq 0
\end{aligned}
$$

for any constant $k$. Since $u_{j}^{n}(x, t)$ is a step function in $x$, the integration with respect to $x$ can be approximated by a Riemann sum of $u_{i, j}^{n+1 / 2}$. Therefore, 
for any small $\varepsilon>0$ we may find a corresponding $\eta$ such that

$$
\begin{aligned}
& \int_{n \Delta t}^{(n+1) \Delta t} {\left[\sum_{i} \phi(i \Delta x, j \Delta y, t)_{t}\left|u_{i, j}^{n+1 / 2}-k\right|\right.} \\
&\left.+\phi_{i, j}(t)_{x} \operatorname{sign}\left(u_{i, j}^{n+1 / 2}-k\right)\left(f_{i, j}^{n+1 / 2}-f(k)\right) \Delta x\right] d t \\
&-\sum_{i} \phi_{i, j}^{n+1}\left|u_{i, j}^{n+1 / 2}-k\right| \Delta x+\sum_{i} \phi_{i, j}^{n}\left|u_{i, j}^{n}-k\right| \Delta x>-\varepsilon,
\end{aligned}
$$

where $f_{i, j}^{n}=f\left(u_{i, j}^{n}\right)$ and $\phi_{i, j}^{n}=\phi(i \Delta x, j \Delta y, n \Delta t)$. Here we have used (1.8) when replacing $u_{j}^{n}(x, \Delta t)$ by $u_{j}^{n+1 / 2}$ and $f_{\delta}\left(u_{j}^{n}(x, \Delta t)\right)$ by $f\left(u_{j}^{n+1 / 2}\right)$. Furthermore, we can approximate differentiation with respect to $t$ by a difference, and integration with respect to $t$ by a multiplication with $\Delta t$. Thus, for any $\varepsilon_{1}>0$ we can find $\eta$ such that

$$
\begin{aligned}
& \sum_{i}\left\{\frac{\phi_{i, j}^{n+1}-\phi_{i, j}^{n}}{\Delta t}\left|u_{i, j}^{n+1 / 2}-k\right|\right. \\
& \left.\quad+\left(\phi_{i, j}^{n+1}\right)_{x} \operatorname{sign}\left(u_{i, j}^{n+1 / 2}-k\right)\left(f_{i, j}^{n+1 / 2}-f(k)\right)\right\} \Delta x \Delta t \\
& \quad-\sum_{i} \phi_{i, j}^{n+1}\left|u_{i, j}^{n+1 / 2}-k\right| \Delta x+\sum_{i} \phi_{i, j}^{n}\left|u_{i, j}^{n}-k\right| \Delta x>-\varepsilon_{1} .
\end{aligned}
$$

Similarly, we get

$$
\begin{aligned}
\sum_{j}\left\{\frac{\phi_{i, j}^{n+1}-\phi_{i, j}^{n}}{\Delta t}\left|u_{i, j}^{n+1 / 2}-k\right|\right. \\
\left.\quad+\left(\phi_{i, j}^{n+1}\right)_{y} \operatorname{sign}\left(u_{i, j}^{n+1 / 2}-k\right)\left(g_{i, j}^{n+1 / 2}-g(k)\right)\right\} \Delta y \Delta t \\
\quad-\sum_{j} \phi_{i, j}^{n+1}\left|u_{i, j}^{n+1}-k\right| \Delta y+\sum_{i} \phi_{i, j}^{n}\left|u_{i, j}^{n+1 / 2}-k\right| \Delta y>-\varepsilon_{2}
\end{aligned}
$$

for any $\varepsilon_{2}>0$ and for some sufficiently small $\eta$. Multiplying (2.22) by $\Delta y$ and adding for all $j$, and multiplying (2.23) by $\Delta x$ and adding for all $i$, and finally adding the results, we get

$$
\sum_{i, j}\left\{\frac{\phi_{i, j}^{n+1}-\phi_{i, j}^{n}}{\Delta t}\left|u_{i, j}^{n+1 / 2}-k\right|+\operatorname{sign}\left(u_{i, j}^{n+1 / 2}-k\right)\right.
$$

$$
\begin{aligned}
& \left.\quad \times\left(\left(\phi_{i, j}^{n+1}\right)_{x}\left(f_{i, j}^{n+1 / 2}-f(k)\right)+\left(\phi_{i, j}^{n+1}\right)_{y}\left(g_{i, j}^{n+1 / 2}-g(k)\right)\right)\right\} \Delta x \Delta y \Delta t \\
& -\sum_{i, j} \phi_{i, j}^{n+1}\left|u_{i, j}^{n+1}-k\right| \Delta x \Delta \\
& +\sum_{i, j} \phi_{i, j}^{n}\left|u_{i, j}^{n}-k\right| \Delta x \Delta y>-L\left(\varepsilon_{1}+\varepsilon_{2}\right)=-L \varepsilon,
\end{aligned}
$$

where $L=N \Delta x=N \Delta y$, and $N$ is such that $\operatorname{supp}(\phi) \subset\{|x|<N / 2,|y|<$ $N / 2\} \times[0, T]$. Summing (2.24) over $n$ and letting $\eta \rightarrow 0$, we get that $u$ is an entropy weak solution of $(2.1)$. 
The generalization of this to higher dimensions is straightforward. We define

$$
G_{\delta}(t)=\pi S_{\delta}^{f_{n}, x_{n}} \cdots \pi S_{\delta}^{f_{1}, x_{1}}
$$

and let $\eta$ denote the 'grid spacing', i.e., $\eta=\left(\delta, \Delta x_{1}, \ldots, \Delta x_{n}, \Delta t\right)$. The approximate solution is denoted

$$
u_{\eta}\left(x_{1}, \ldots, x_{n}, m \Delta t\right)=\left(G_{\delta}(\Delta t)\right)^{m} u_{0}\left(x_{1}, \ldots, x_{n}\right) .
$$

Theorem. Let $f_{1}, \ldots, f_{n}$ be continuous functions that are in $B V_{\text {loc }}(\mathbb{R}) \cap L_{\text {loc }}^{1}(\mathbb{R})$. Define by (2.26) a sequence of approximate solutions of $(0.1)$ indexed by $\eta$. As $\eta \rightarrow 0$, a subsequence of $u_{\eta}$ converges to the unique entropy weak solution (0.2) of $(0.1)$.

\section{TWO NUMERICAL EXAMPLES}

In this section we will present two numerical examples where the method of fractional steps has been applied. The first is a theoretical example, while the second is taken from petroleum reservoir simulation.

In the first example we study the equation

$$
\begin{aligned}
u_{t}+\left(u^{2}\right)_{x}+\cos (2 \pi u)_{y}=0, \\
u_{0}(x, y)= \begin{cases}-1 & \text { if }|(x, y)-(0.4,0.4)|<0.5, \\
1 & \text { if }|(x, y)-(-0.4,-0.4)|<0.5, \\
0 & \text { otherwise }\end{cases}
\end{aligned}
$$

We have used $\Delta x=\Delta y=.05$ and $\Delta t=0.10$, which corresponds to a CFL number of 4 in the $x$-direction and 2 in the $y$-direction. We have used 11 piecewise linear segments to approximate the flux functions. As the measure $\mu$ we have here used simple Lebesgue measure, and the projection $\pi$ from (1.9) is therefore simply the grid cell average. In Figures 3.1-3.4 (see next page) we show the solution at times $t=0, t=0.1, t=0.2$ and $t=0.3$, respectively. The different values are shown on a gray scale such that black corresponds to -1 and white corresponds to 1 . The labels on the axes denote grid blocks, the lower left-hand corner has coordinates $(-1,-1)$ and the upper right-hand corner $(1,1)$.

The next example is taken from petroleum reservoir simulation. It is a study of water injection into a homogeneous horizontal oil field. We use a two-phase black oil model, neglecting capillary pressure, and assume that the two phases present, water and oil, are incompressible. The reservoir is then described by two coupled differential equations called 'pressure equation' and 'saturation equation', respectively. The pressure equation reads

$$
\nabla\left(\left(\lambda_{o}(s)+\lambda_{w}(s)\right) \nabla P\right)=0 .
$$

Here, the unknowns are the pressure $P$, the fraction of the available pore volume occupied by water, and the water saturation $s$. The relative permeabilities $\lambda_{o}(s)$ and $\lambda_{w}(s)$ are assumed to be known functions of the saturation. By using the so-called Darcy's law, one obtains the saturation equation

$$
s_{t}+\nabla F(s)=0
$$

where

$$
F(s)=\frac{\lambda_{w}(s)}{\lambda_{o}(s)+\lambda_{w}(s)} \nabla P
$$




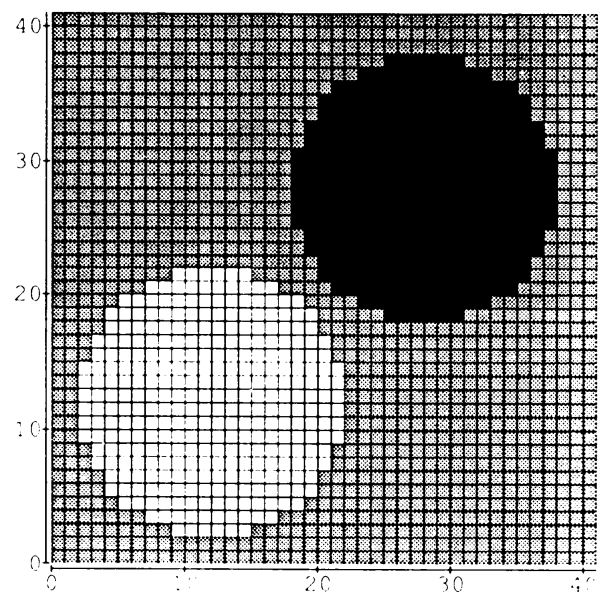

FIGURE 3.1

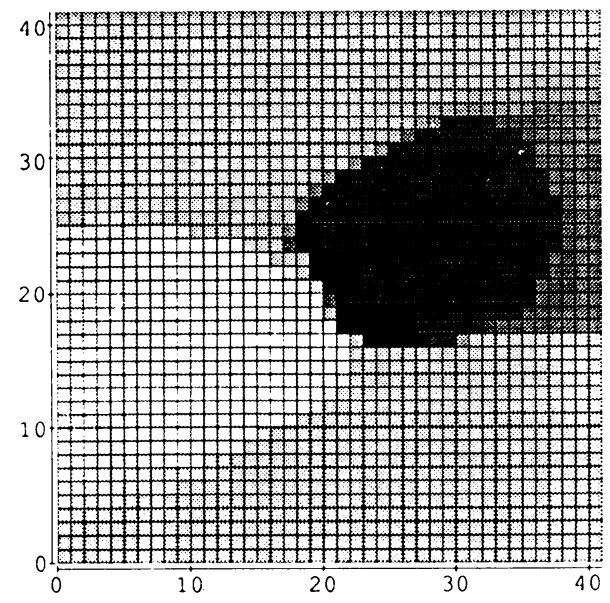

FIGURE 3.3

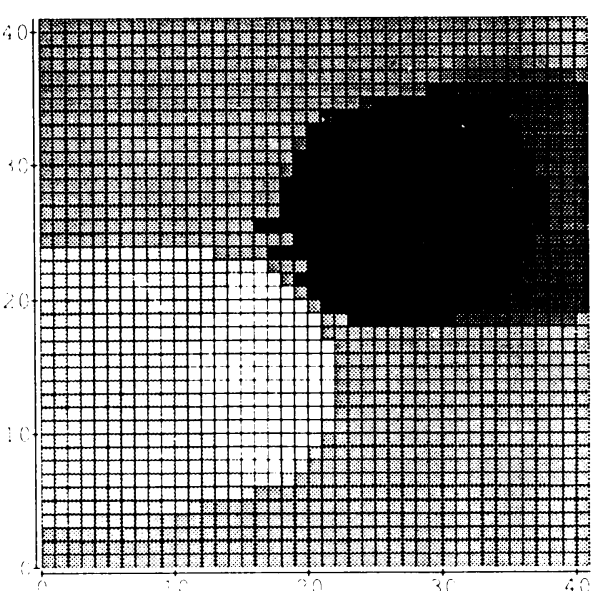

FIGURE 3.2

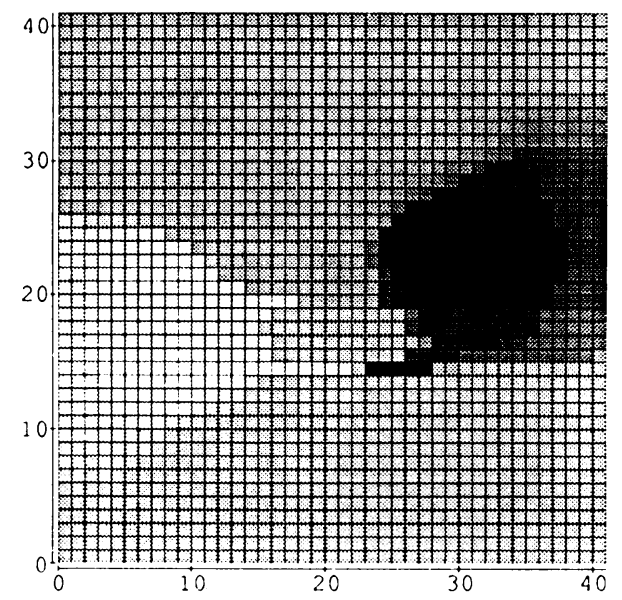

FIGURE 3.4

The equations (3.2) and (3.3) are solved using an IMPES method, i.e., first (3.2) is solved with the initial saturation distribution, then (3.3) is solved in some time interval $\left[t_{0}, t_{1}\right]$, assuming that the pressure is constant in this interval. Now we again solve (3.2), given the result from (3.3). This process is then repeated. The IMPES method is common, and is usually justified by the large difference in the characteristic times in (3.2) and (3.3). The numerical method used to solve (3.3) is a finite element method using the same grid system as the method of fractional steps which is used to solve (3.4). Here the projection $\pi$ is much more sophisticated than in the theoretical example. The algorithm for $\pi$ 'tries to discover' where the solution is smooth and where it is discontinuous. In places where it finds discontinuities it tries to represent these as well as possible. 

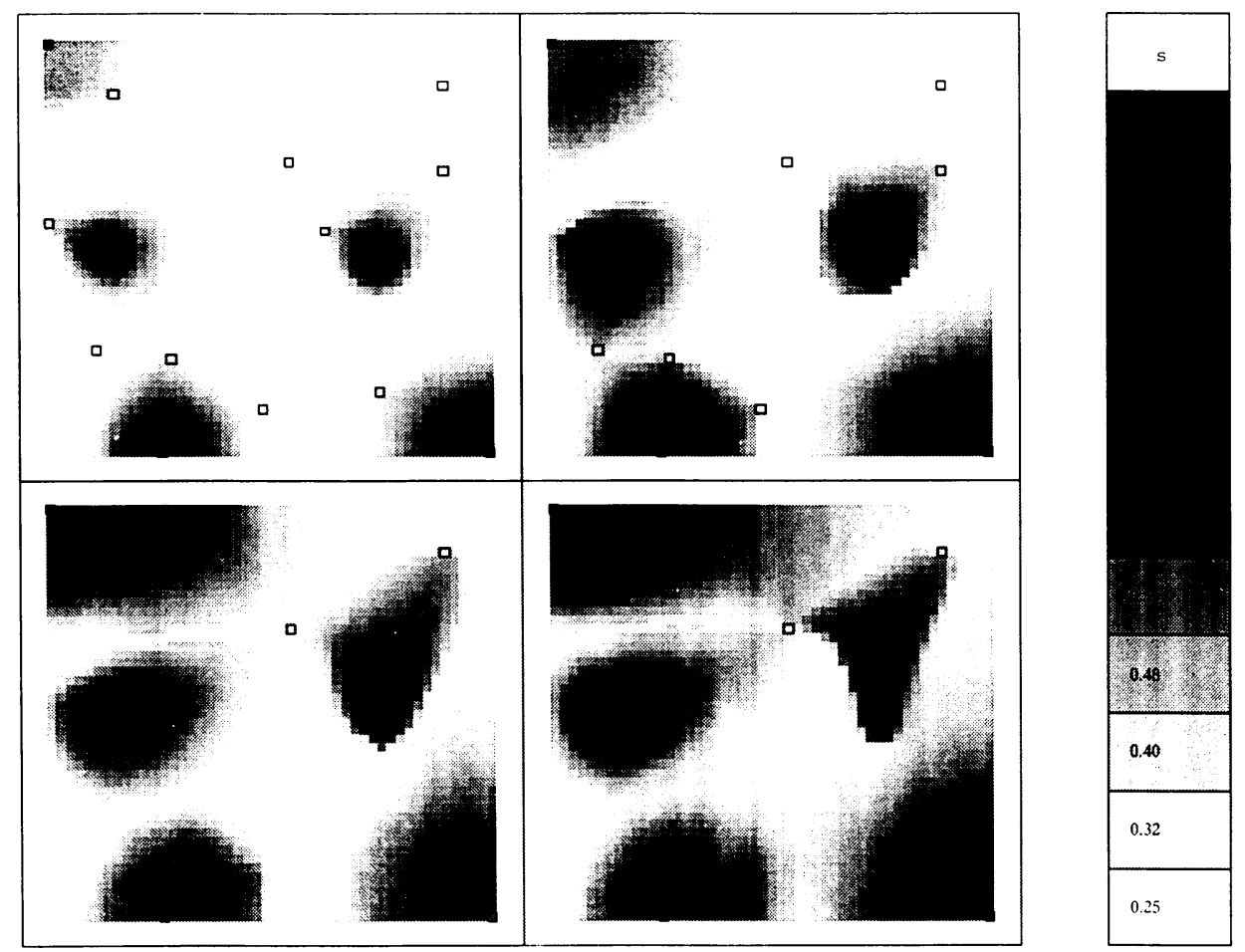

FIGURE 3.5

For further details of the fractional steps algorithm applied to flow in porous media the reader is referred to [1].

In the example we use five injection wells and initially ten production wells. A production well is automatically shut off after water breakthrough, i.e., after the saturation surrounding it has reached some predefined level. In Figure 3.5 we show the saturation of water at four different times, all active wells are marked by squares, for injection wells the squares are black while production wells are white. The water saturation is represented by a gray scale, black corresponds to a saturation of 1.0 and white to a saturation of 0.25 . The numerical grid size used here is $50 \times 50$, and we used ten linear segments in the approximation to the flux function.

\section{BIBLIOGRAPHY}

1. F. Bratvedt, K. Bratvedt, C. Buchholz, T. Gimse, H. Holden, L. Holden, and N. H. Risebro, Front tracking for petroleum reservoirs, in Ideas and Methods in Mathematics and Physics (S. Albeverio, J. E. Fenstad, H. Holden, T. Lindstrøm, eds.), Cambridge Univ. Press, Cambridge, 1992, pp. 409-427.

2. E. Conway and J. Smoller, Global solutions of the Cauchy problem for quasilinear first order equations in several space variables, Comm. Pure Appl. Math. 19 (1966), 95-105.

3. M. Crandall and A. Majda, The method of fractional steps for conservation laws, Numer. Math. 34 (1980), 285-314.

4. C. M. Dafermos, Polygonal approximations of solutions of the initial value problem for $a$ conservation law, J. Math. Anal. Appl. 38 (1972), 33-41.

5. S. K. Godunov, Finite difference methods for numerical computations of discontinuous solutions of the equations of fluid dynamics, Mat. Sb. 47 (1959), 271-306 (Russian). 
6. H. Holden and L. Holden, On scalar conservation laws in one dimension, in Ideas and Methods in Mathematics and Physics (S. Albeverio, J.E. Fenstad, H. Holden, T. Lindstrøm, eds.), Cambridge Univ. Press, Cambridge, 1992, pp. 480-509.

7. H. Holden, L. Holden, and R. Høegh-Krohn, A numerical method for first order nonlinear scalar conservation laws in one dimension, Comput. Math. Appl. 15 (1988), 595-602.

8. S. N. Kružkov, First order quasilinear equations in several independent variables, Math. USSR-Sb. 10 (1970), 217-243.

9. N. Kuznetsov, Weak solution of the Cauchy problem for a multi-dimensional quasi-linear equation, Math. Notes 2 (1967), 733-739.

10. A. I. Vol'pert, The spaces $B V$ and quasilinear equations, Math. USSR-Sb. 2 (1967), 225-267.

Division of Mathematical Sciences, The norwegian Institute of Technology, The UNIVERSITY OF TRONDHEIM, N-7034 TRONDHEIM, NORWAY

E-mail address: holden@imf.unit.no

Department of Mathematics, University of Oslo, P.O. Box 1053, Blindern, N-0316 OsLO, NORWAY

E-mail address: nilshr@ikaros.uio.no 